\title{
Generalized Granuloma Annulare Can Be Presented With Different Clinical Morphologies in the Same Patient: A Case Report
}

\author{
Ayman Mahran ${ }^{1 *}$, Mohamed Allam¹, Hiba Ahmed ${ }^{1}$, Alaa Ghazally', Asmaa M Ahmed ${ }^{2}$ \\ ${ }^{1}$ Dermatology, Venereology and Andrology Department, Faculty of Medicine, Assiut University, Assiut, Egypt. \\ ${ }^{2}$ Pathology Department, Faculty of Medicine, Assiut University, Assiut, Egypt. \\ *Corresponding Author: Ayman Mahran, Dermatology, Venereology and Andrology Department, Faculty of Medicine, Assiut University, \\ Assiut, Egypt E-mail: aymanderma@yahoo.com \\ Received date: October 12, 2019: Accepted date: October 23, 2019: Published date: October 30, 2019 \\ Citation: Ayman Mahran, (2019) Generalized Granuloma Annulare Can Be Presented With Different Clinical Morphologies In The Same \\ Patient: A Case Report 4(2) DOI: 10.31579/2578-8949/051
}

Copyright: (02019. Ayman Mahran This is an open-access article distributed under the terms of the Creative Commons Attribution License, which permits unrestricted use, distribution, and reproduction in any medium, provided the original author and source are cre dited.

\begin{abstract}
The term granuloma annulare (GA) appropriately describes the classic type characterized by ringed erythematous plaques with histological palisaded granulomatous inflammation. However, GA now includes a range of disease. Over time, more atypical, rare types have been reported in isolated case reports or small case series. Here, we describe a unique case of generalized GA presented by two morphologically different lesions in a 52 years old diabetic man. To the best of our knowledge, this association has never been reported in the literature.
\end{abstract}

Keywords: generalized granuloma annulare, atypical granuloma annulare

\section{Introduction}

Granuloma annulare is considered a noninfectious granulomatous skin condition.[1].GA can be presented by multiple clinical types; including localized, generalized, subcutaneous, perforating and many atypical morphologies[2]. Histologically, GA shows collagen degeneration, mucin deposition, and either a palisaded or interstitial histiocytic infiltrate[3].Although the exact etiology is unknown, a cell-mediated hypersensitivity reaction is suggested[4].There are multiple reports of systemic associations with GA, including diabetes mellitus (DM), thyroid dysfunction, malignancy, lipid abnormalities, and infection[5].

\section{CASE:}

A fifty two years old man presented to our clinic by asymptomatic skin lesions over the neck and extremities of 5 months duration. It started on the forearms then spreaded to the neck one month later and gradually increased in size and number. The medical condition of the patient is unremarkable except for diabetes mellitus which was diagnosed four years earlier. Dermatological examination revealed multiple erythematous infiltrated plaques symmetrically distributed over the dorsal aspect of both forearms and hands. (figure 1).

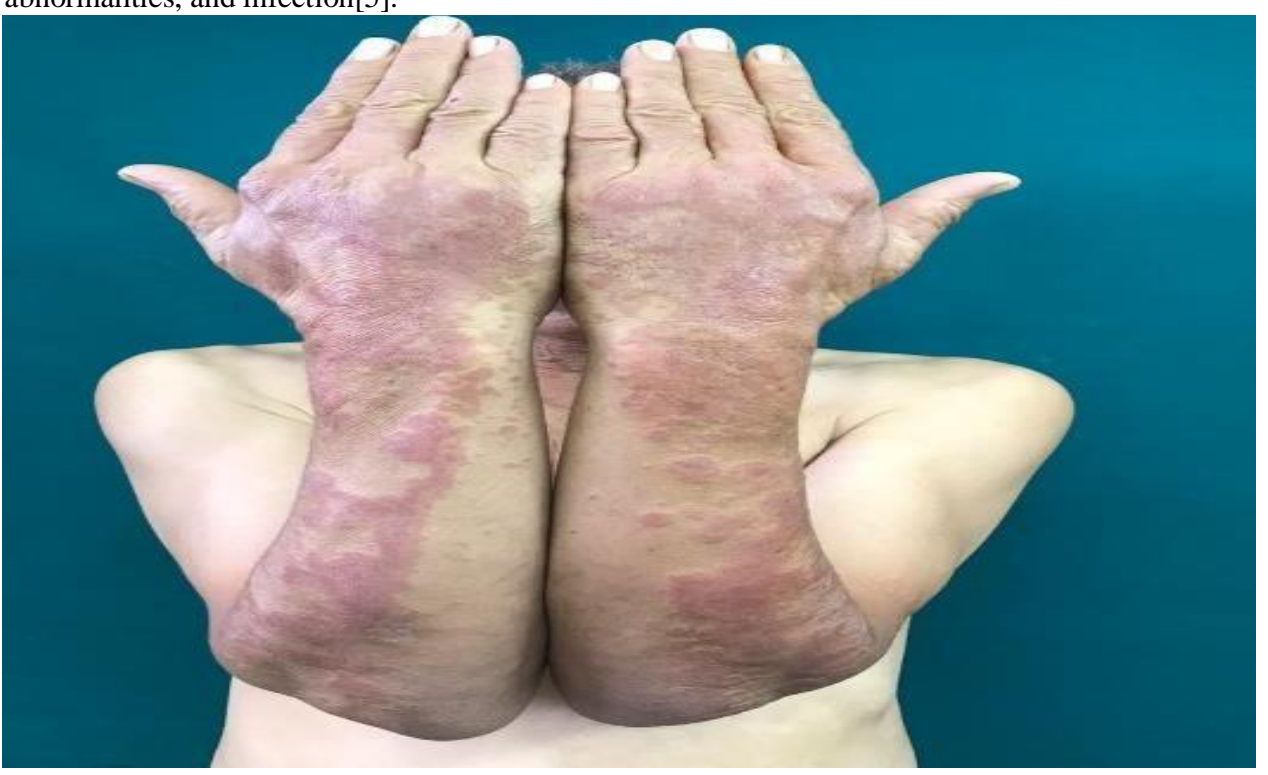

Figure 1: multiple infiltrated erythematous plaques over the dorsal aspects of both forearms and hands. 
Another lesions of different morphology composed of flesh-colored to erythematous papules, some of them are discrete and others are coalescing into annular plaques over the neck and upper part of the chest (figure 2).
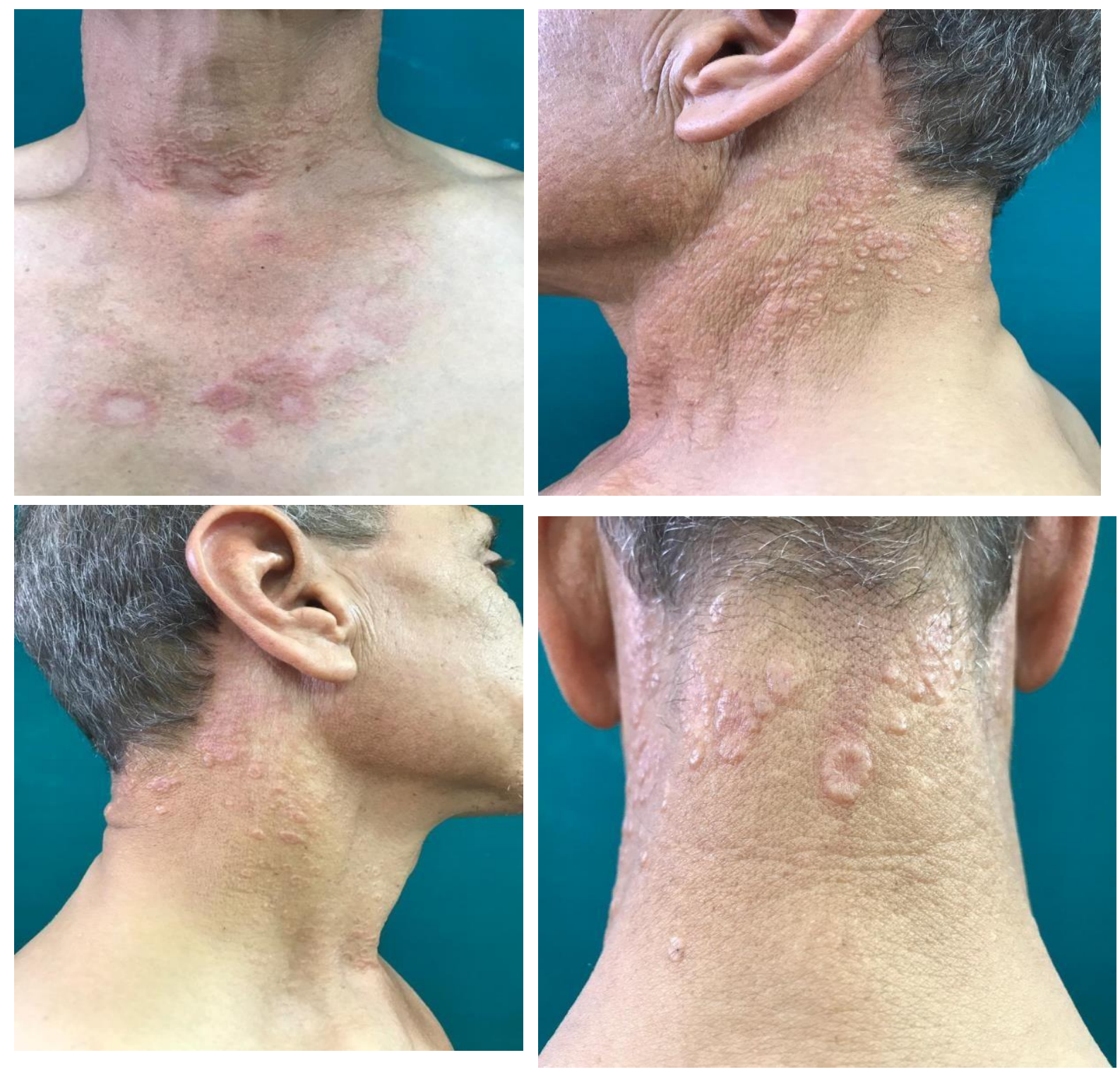

Figure 2: multiple papules and annular plaques over the neck and upper chest.

Routine and relevant laboratory investigations were normal except for elevated random blood sugar level: $12.5 \mathrm{mmol} / \mathrm{L}$ (normal value: 4-7.8 $\mathrm{mmol} / \mathrm{L}$ ). Two skin biopsies were obtained (one from annular lesions over the neck and the other from infiltrated plaque over the forearms) which revealed almost similar findings in both with the presence of areas of degenerated (necrobiotic) collagen, surrounded by palisaded infiltrate of histiocytes and fibroblasts with few multinucleated giant cells and moderate perivascular and interstitial lymphocytic infiltrate in the surrounding dermis. (figure 3 and 4). 


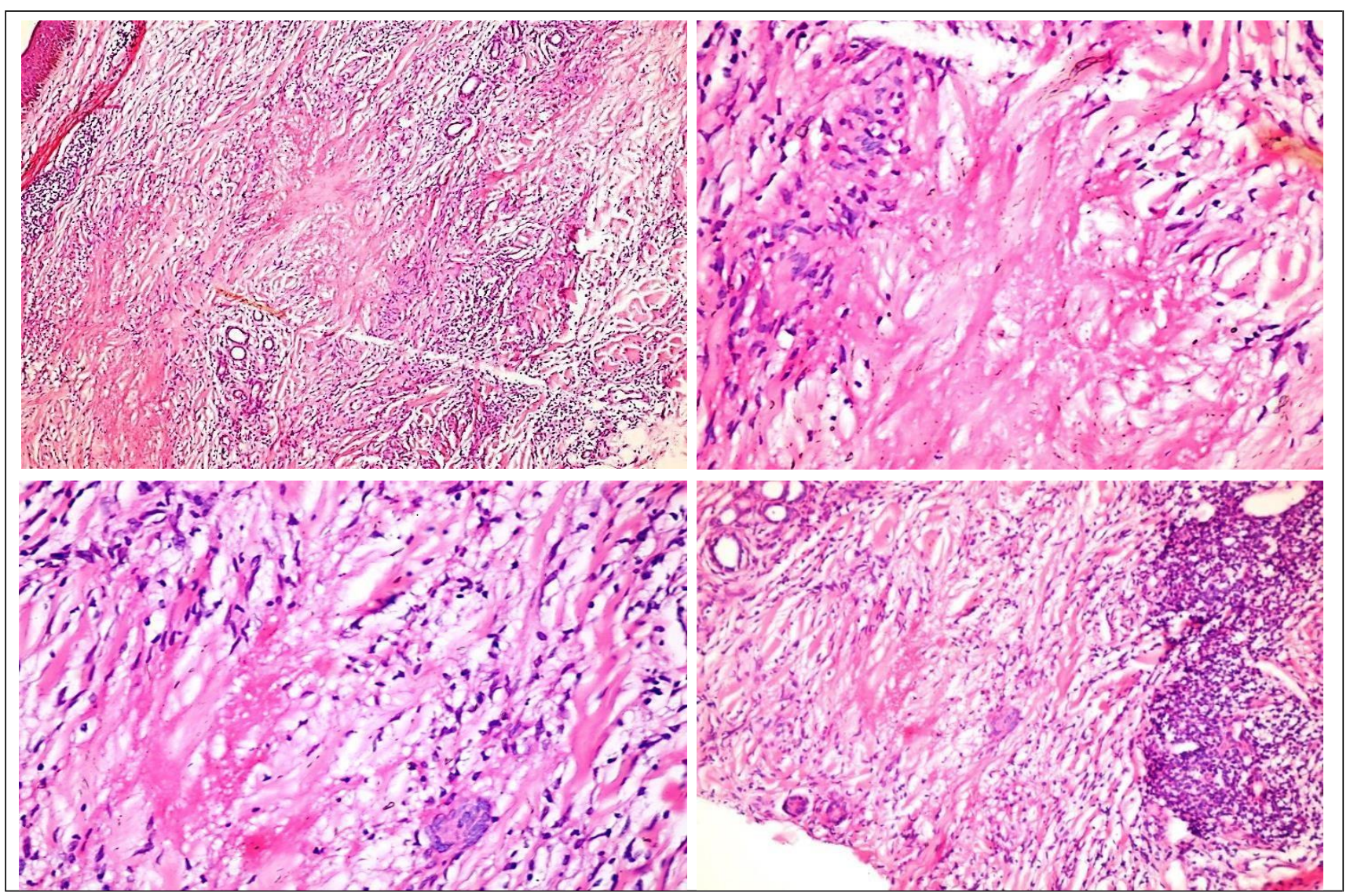

Figure3: Microscopic examination revealed: A) Areas of degenerated (necrobiotic) collagen (arrow), x200. B) Palisaded infiltrate of histiocytes and fibroblasts surrounding the degenerated collagen, $x 400$. C) Few multinucleated giant cells, $x 400$. D) Moderate perivascular and interstitial lymphocytic infiltrate in the surrounding dermis, $x 400$.

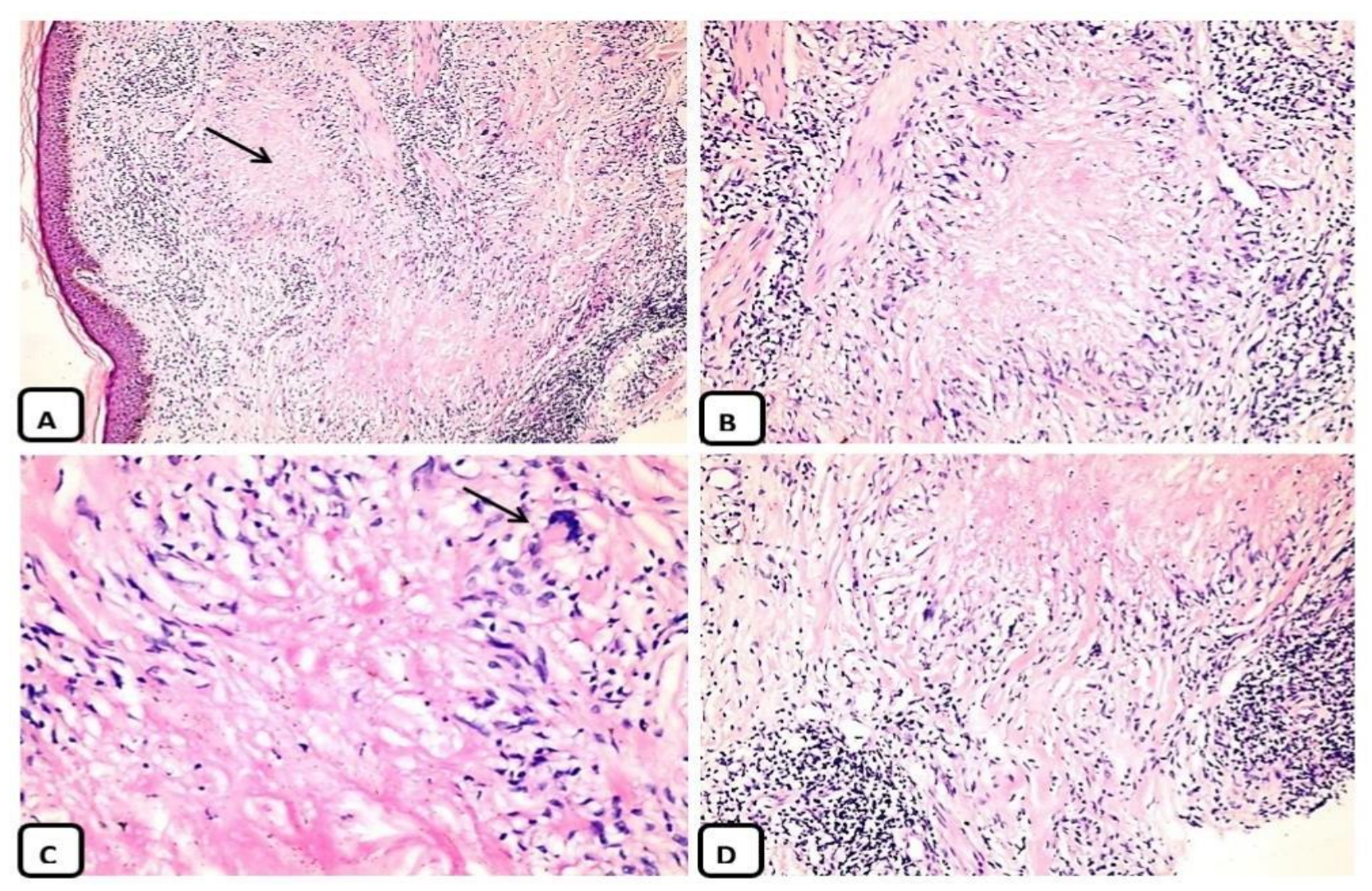




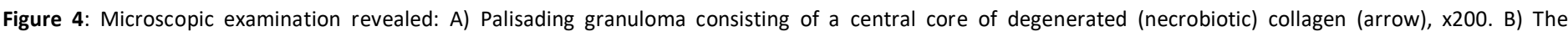

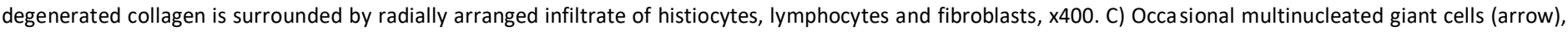
$x 400$. D) Moderate perivascular and interstitial lymphocytic infiltrate in the surrounding dermis, $x 400$.

\section{Discussion}

Here we present a case of 52 -year-old diabetic man presented with two clinical morphologies. The lesions over the neck and upper part of the chest are identical to the classic annular presentation of GA with strict photosensitive distribution. On the other hand, the lesions over the forearms and hands are composed of multiple large infiltrated plaques which is an unusual presentation of GA. Although many reports described cases of generalized GA presented with plaques [6] and others reported cases with photosensitive distribution,[7]. none described the coexistence of such two variants (annular and infiltrated plaques) like in our case.

The differential diagnosis for the neck lesions revolves around GA, actinic granuloma, sarcoidosis and porokeratosis. While, the differential diagnosis for the upper limb lesions included GA, leprosy, sarcoidosis and mycosis fungoides. Histological examination of two biopsies from two different lesions confirmed typical feature of GA. We considered this case as a case of generalized GA with atypical presentation.

Granuloma annulare is a benign inflammatory skin disorder with varying morphologies, and multiple subtypes [5].Generally, the age incidence is between the $3^{\text {rd }}$ and $5^{\text {th }}$ decades of life, with a female to male ratio of about 1-2:1[8,9]. The most common subtype is localized GA, occurring in $75 \%$ of cases[10].Generalized GA is the second most common variant, it occurs in about $15 \%$ of patients[11]. Whereas localized GA affects young adult females, generalized GA is slightly more common in males with two peak age incidences, children under 10 years and adults over 40 years[12]. Both age and sex incidences perfectly correlate with our case.

Granuloma annulare may be idiopathic, but many studies reported associations with many systemic conditions. Diabetes is one of the most reported diseases associated with GA,[13] which is the case in our patient[5].

Localized GA is usually asymptomatic and self-resolving within two years, however, the patient may need treatment for cosmetic reasons. Treatment is often challenging for generalized GA, because of its recalcitrant nature and a paucity of evidence-based therapy. Many different treatment modalities have been described for GA with different results. These include topical potent corticosteroids, intralesional triamcinolone, topical calcineurin inhibitors, dapsone 5\% gel and topical vitamin E. Systemic therapies include dapsone, doxycycline, retinoids, antimalarials, nicotinamide and infliximab. Phototherapy may also be helpful.[10].

The new clinical remark about GA which was noted for the first time in the literature in our case is: Generalized GA can be presented by different clinical morphologies (annular and infiltrated plaques) in the same patient which may create confusion and delay in the diagnosis, and, hence appropriate management.

\section{References:}

1. Studer EM, Calza AM, Saurat JH. (1996) Precipitating factors and associated diseases in 84 patients with GA: a retrospective study. Dermatology 1996;193(4):364-368.

2. Piette EW, Rosenbach M.( 2016) GA: clinical and histologic variants, epidemiology, and genetics. J Am Acad Dermatol;75:457-465.

3. Umbert P, Winkelmann RK.(1977) Histologic, ultrastructural and histochemical studies of GA. Arch Dermatol;113(12):1681-1686.

4. Buechner SA, Winkelmann RK, Banks PM. (1983) Identification of Tcell subpopulations in GA. Arch Dermatol;119(2):125-128.

5. Keimig EL.( 2015) GA. Dermatol Clin.;33(3):315329.

6. Fujimoto N, Manabe T, Tanaka T. GA (2018) presenting with a giant plaque, successfully treated with minocycline. Eur J Dermatol. Oct 1;28(5):696-697

7. Gass JK, Todd PM, Rytina E.( 2009) Generalized GA in a photosensitive distribution resolving with scarring and milia formation. Clin Exp Dermatol;34(5):e53-55.

8. Muhlbauer JE. GA. J Am Acad Dermatol. 1980;3:217-230.

9. Thornsberry LA, (2013) English JC 3rd. Etiology, diagnosis, and therapeutic management of GA: an update. Am J Clin Dermatol.;14:279290.

10. Cyr PR. Diagnosis and management of GA. Am Fam Physician. 2006;74(10):1729-1734.

11. Lukacs J, Schliemann S, Elsner P. (. 2015) Treatment of generalized GA -a systematic review. J Eur Acad Dermatol Venereol;29(8):14671480 .

12. Yun JH, Lee JY, Kim MK, Seo YJ, Kim MH, et al. (2009) Clinical and pathological features of generalized GA with their correlation: a retrospective multicenter study in Korea. Ann Dermatol.;21(2):113-119. 13. Piette EW, Rosenbach M. GA: pathogenesis, disease associations and triggers, and therapeutic options. J Am Acad Dermatol. 